OPEN ACCESS

Edited by:

Andrew W. Taylor,

Boston University, United States

Reviewed by:

Carlos Cabañas,

Spanish National Research Council

(CSIC), Spain

*Correspondence:

Gongping Deng

denggongping@hainmc.edu.cn

Shan Zeng

zengshan2000@csu.edu.cn

Specialty section:

This article was submitted to Immunological Tolerance and Regulation,

a section of the journal

Frontiers in Immunology

Received: 11 November 2021

Accepted: 12 January 2022

Published: 31 January 2022

Citation:

Tang D, Liu S, Shen H, Deng G and Zeng S (2022) Extracellular Vesicles

Promote the Formation of PreMetastasis Niche in Gastric Cancer.

Front. Immunol. 13:813015. doi: 10.3389/fimmu.2022.813015

\section{Extracellular Vesicles Promote the Formation of Pre-Metastasis Niche in Gastric Cancer}

\author{
Diya Tang ${ }^{1,2}$, Shanshan Liu ${ }^{1,2}$, Hong Shen ${ }^{2}$, Gongping Deng ${ }^{3 *}$ and Shan Zeng ${ }^{1 *}$ \\ ${ }^{1}$ Department of Oncology, Xiangya Hospital, Central South University, Changsha, China, ${ }^{2}$ National Clinical Research Center \\ for Geriatric Disorders, Xiangya Hospital, Central South University, Changsha, China, ${ }^{3}$ Department of Emergency, Hainan \\ General Hospital, Hainan Affiliated Hospital of Hainan Medical University, Haikou, China
}

Globally, gastric cancer (GC) ranks fourth in the incidence of malignant tumors. The early clinical manifestations of GC lack specificity. Most patients are already at an advanced stage when they are first diagnosed, and their late progression is mainly due to peritoneal metastasis. A pre-metastatic microenvironment is formed, before the macroscopic tumor metastasis. Extracellular vesicles (EVs) are nanovesicles released by cells into body fluids. Recent studies have shown that EVs can affect the tumor microenvironment by carrying cargos to participate in cell-to-cell communication. EVs derived from GC cells mediate the regulation of the pre-metastasis niche and act as a coordinator between tumor cells and normal stroma, immune cells, inflammatory cells, and tumor fibroblasts to promote tumor growth and metastasis. This review highlights the regulatory role of EVs in the premetastatic niche of GC and mulls EVs as a potential biomarker for liquid biopsy.

Keywords: gastric cancer, extracellular vesicles, immunomodulation, pre-metastatic, microenvironment

\section{INTRODUCTION}

Gastric cancer (GC) is one of the major global health problems. There are about one million new cases of GC reported all over the world yearly $(1,2)$. Most GC patients present with locally advanced or distant metastasis with an average overall survival of 10-12 months (3). Stage IIIC resected tumors carry a mere 5 -year survival rate of $18 \%$. Instead, the 5-year survival rates for stage IA and IB tumors treated with surgery are $94 \%$ and $88 \%$, respectively (4). The pre-metastasis niche is a favorable microenvironment created by the primary tumor for the subsequent metastatic organs,

\footnotetext{
Abbreviations: ApoE, Apolipoprotein E; BMP, Bone morphogenetic proteins; BLID, BH3-like motif-containing protein; CAF, Cancer-associated fibroblasts; CD97, Recombinant Cluster Of Differentiation 97; CDH1, Cadherin-1; EGFR, Epidermal growth factor receptor; EphA2, Erythropoietin-producing hepatocellular A2; EMT, Epithelial-mesenchymal transition; ESCRT, The endosomal sorting complex required for transport; EVs, Extracellular vesicles; FOXQ1, Forkhead box Q1; FZD10, Frizzled10; GC, Gastric cancer; HGF, Hepatocyte growth factor; HOTTIP, The HOXA transcript at the distal tip may; HUVECs, Human umbilical vein endothelial cells; HMGA2, High mobility group AT-hook 2; IVL, Intraluminal vesicle; MIA, Melanoma inhibitory activity; MMT, Mesothelial-to-mesenchymal transition; MVBs, Multivesicular body; MAPK, Mitogenactivated protein kinase; mTOR, Mammalian target of rapamycin; nSMase, Lipid metabolism enzymes neutral sphingomyelinase; PMCs, Peritoneal mesothelial cells; PM, Peritoneal metastasis; PTEN, Phosphatase and tensin homolog; RHBDF2, Rhomboid 5 Homolog 2; SUFU, Suppressor of the fused protein; SFRP1, Secreted frizzled-related protein 1; TAMs, Tumor-associated macrophages; TGF- $\beta$, Transforming growth factor $\beta$; TGF- $\beta 1$, Transforming growth factor $\beta 1$; Treg, Regulatory T; VEGF, Vascular endothelial growth factor; Wnt, Wingless/Integrated.
} 
following the famous "soil" and "seed" metastasis theory (5-7). According to the difference of biogenesis, EVs can be divided into microvesicles and exosomes. Their contents include nucleic acids, lipids, and proteins which can promote the malignant progression of cancer cells (8). GC-derived EVs play a critical role in tumor formation, proliferation, invasion, metastasis, drug resistance, and other processes. This article addresses the role of GC-derived EVs in the formation of pre-metastasis niches in GC.

\section{BIOGENESIS OF EVS}

In a stable external environment, EVs can be released by all organisms (9). EVs are heterogeneous groups that, according to their biological origin, can be divided into two types: microvesicles and exosomes (10). The biogenesis, cargo assembly, secretion of exosome involves a set sequence of cellular events (Figure 1). Exosomes are nano-sized vesicles formed by early endosomes sprouting inward, leading to the generation and enrichment of intraluminal vesicles. It selectively contains proteins, nucleic acids, and lipids used to form advanced endosomes - multi vesicles body (MVBs). MVBs can be integrated with the plasma membrane, freeing the jammed intraluminal vesicle (ILV) (So-called exosomes) (10, 11). The endosomal sorting complex required for transport (ESCRT) is the chief driver of membrane shaping and splitting, and the conventional mechanism of MVBs and ILV formation $(12,13)$. ESCRT comprises four dissimilar protein complexes; ESCRT-0, -I, -II, -III, and auxiliary protein $(14,15)$. The utmost comprehensive research of ESCRTs in exosome biogenesis demonstrated that four in twenty-three ESCRT proteins affected exosomes' secretion, and they include; HRS, TSG101, STAM1, and VPS4B protein (16). Interestingly, despite concurrent silencing of vital subunits of all four ESCRTcomplexes, ILVs are still molded in MVBs (17). Therefore, it is possible to assume that exosomes can also be developed in an ESCRT-independent manner, like the tetraspanin family (CD63, CD81, CD83) and lipid metabolism enzymes neutral sphingomyelinase (nSMase) (10). Compared to the biological generation of exosomes, microvesicles are somewhat more straightforward. New microvesicles are formed by the outward budding and contraction of the plasma membrane (11).

\section{GC-DERIVED EVs}

Kagota et al. combined pretreatment technology with ultracentrifugation to separate and purify EVs from gastric juice of GC patients (18). The substances released by EVs included DNA, RNA, proteins, lipids, and metabolites (19). Extensive previous research has proved that the protein cargo of EVs can speed up the process of GC metastasis (Table 1) (20$24)$. For example, by detecting the expression of TGF- $\beta 1$ in exosomes isolated from the gastric omental veins of $61 \mathrm{GC}$ patients, as well as regulatory $\mathrm{T}$ cells (Treg) in lymph nodes of GC. The researchers found that the expression of TGF- $\beta 1$ was closely related to the pathological stage, lymph node metastasis, and the proportion of Treg cells. In addition, exosomes derived from GC patients act on TGF- $\beta 1$, which can induce Treg cell formation (25). Ectopic expression of exosomes has been observed, whereby the EGFR expressed by exosomes isolated from GC cells not only enters the liver but also integrates into the plasma membrane of liver stromal cells. Also, translocation of EGFR promoted tumor invasion and metastasis by hindering $\mathrm{miR}-26 \mathrm{a} / \mathrm{b}$, thus, up-regulating the expression of hepatocyte growth factor (HGF) (26).
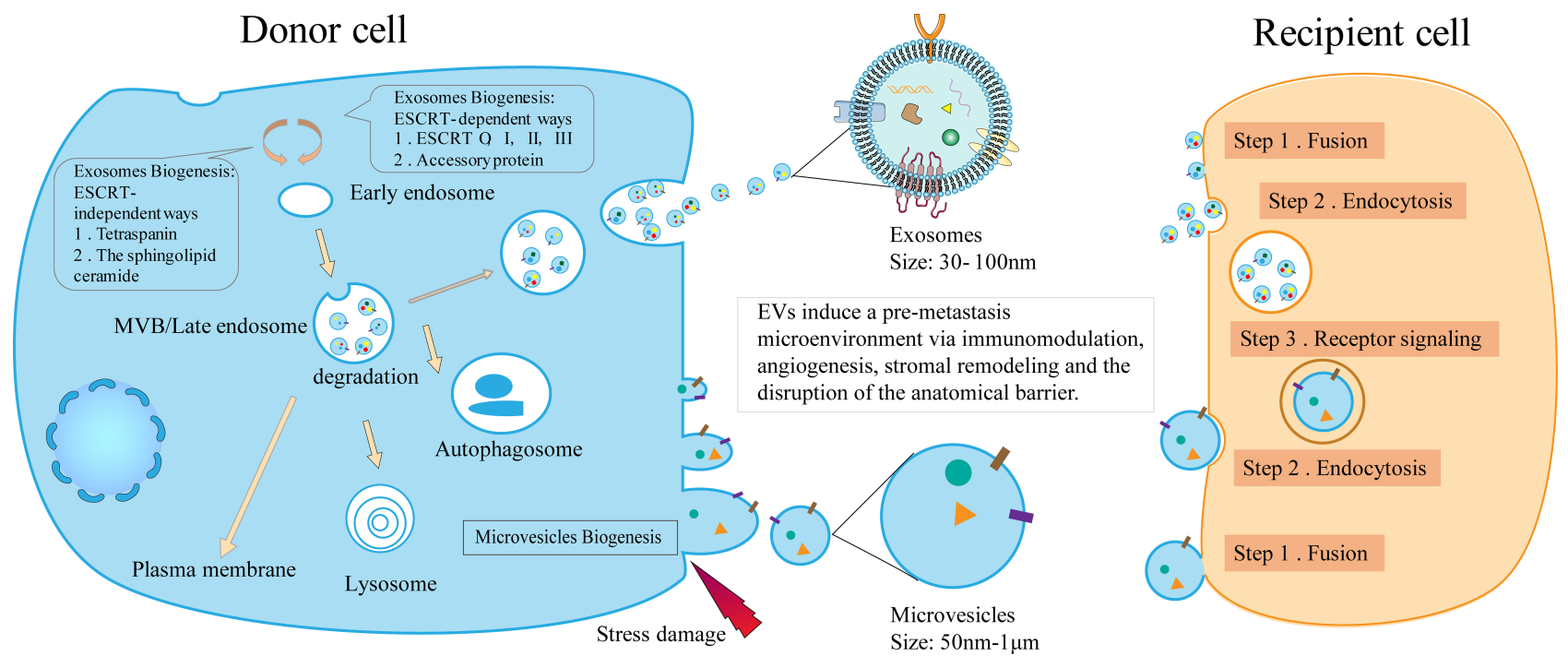

FIGURE 1 | The biogenesis of EVs and the mechanism of the formation of pre-metastasis microenvironment. The biogenesis of EVs includes the occurrence of exosomes and microvesicles. By acting as communicators between donor and recipient cells, EVs induce immunomodulation, angiogenesis, stromal remodeling, and barrier disruption, ultimately contributing to the pre-metastasis microenvironment. 
TABLE 1 | Summary of proteins in EVs involved in GC progression and metastasis.

\begin{tabular}{|c|c|c|}
\hline Extracellular vesicles proteins & Recipient cells/Target & Role/Mechanism \\
\hline CD97 & NA & Lymphatic metastasis (20) \\
\hline CD97 & MAPK signaling pathway & Proliferation and Invasion (21) \\
\hline FZD10 & NA & Carcinogenesis and Tumor proliferation (22) \\
\hline FZD10 & Wnt signaling pathway & Cancer progression (23) \\
\hline MET & TAMs & Cancer progression (24) \\
\hline TGF- $\beta 1$ & Treg cells & Lymphatic metastasis (25) \\
\hline EGFR & HGF & Liver metastasis (26) \\
\hline
\end{tabular}

Evs, Extracellular vesicles; EGFR, Epidermal growth factor receptor; FZD10, Frizzled10; GC, Gastric cancer; HGF, Hepatocyte growth factor; TAMs, Tumor-associated macrophages; Treg, Regulatory T; TGF- $\beta 1$, Transforming growth factor $\beta 1$; Wnt, Wingless/Integrated; MAPK, Mitogen-activated protein kinase; MET, Mesenchymal-epithelial transition factor NA, Not available.

Apart from proteins, nucleic acids also play a vital role in the metastasis of GC. For instance, when exosomes were isolated from GC cells and gastric mucosal epithelial cells, the expression level of miR-155-5p in GC- derived exosomes were significantly increased. Simultaneously, the expression of TP53INP1 protein in GC cells was down-regulated, which confirmed that TP53INP1 was directly affected by miR-155-5p regulation. More importantly, when GC cell line AGS was cultured with isolated exosomes rich in miR-155-5p, the proliferation and migration ability of AGS cells was increased, confirming that exosome miR-155-5p facilitated the invasion and metastasis in GC by directly acting on TP53INP1 (27). In vitro experiments showed that GC-derived exosomes can be absorbed by peritoneal mesothelial cells and further up-regulate the expression of miR21-5p and directly target SMAD7 to promote peritoneal metastasis. The researchers also established a mouse tumor peritoneal diffusion model, verifying that exosome miR-21-5p induces peritoneal mesothelial cells (PMCs) mesothelial-tomesenchymal transition (MMT) and promote cancer to the peritoneum by targeting SMAD7 (28). After co-cultivation of umbilical vein endothelial cells and GC-derived exosomes, the expression of miR-23a was up-regulated and directly targeted PTEN to promote tumor angiogenesis. Furthermore, VEGF was up-regulated, and TSP-1 was down-regulated (29). Collectively, the nucleic acid cargo of EVs regulates the gene expression of recipient cells not only locally but also in a systemic manner. Also, in the initial stage of malignant tumors, it promotes the formation of the pre-metastasis microenvironment and subsequent migration (Table 2) (30-48).

\section{ROLES OF GC-DERIVED EVs IN REMODELING THE PRE-METASTASIS NICHE OF GC}

We summarized the roles of GC-derived EVs in reshaping the pre-metastasis niche of GC which promotes the colonization and

TABLE 2 | Summary of non-coding RNAs in EVs involved in GC progression and metastasis.

\begin{tabular}{|c|c|c|}
\hline Non-coding RNAs & Recipient cells/Target & Role/Mechanism \\
\hline$m i R-21-5 p$ & PMCs & Peritoneal metastasis (28) \\
\hline$m i R-23 a$ & HUVECS/PTEN & Angiogenesis (29) \\
\hline miR-106a & Smad7 & Peritoneal metastasis (31) \\
\hline$m i R-15 b-3 p$ & DYNLT1/Caspase-3/Caspase-9 Signaling Pathway & Tumorigenesis and Malignant transformation (32) \\
\hline miR-155 & Endothelial Cells/c-MYBNEGF Axis & Angiogenesis (33) \\
\hline$m i R-1290$ & NKD1 & Proliferation and Invasion (36) \\
\hline $\mathrm{miR}-135 b$ & Endothelial Cells/FOXO1 Expression & Angiogenesis (37) \\
\hline miR-501 & BLID & Chemoresistance and Tumorigenesis (38) \\
\hline miRNA expression profiles analysis: 29 miRNA were identified & NA & Peritoneal dissemination (39) \\
\hline miR-423-5p & SUFU & Cancer growth and Metastasis (40) \\
\hline $\mathrm{miR}-130 \mathrm{a}$ & Vascular endothelial Cells/Targeting C-MYB & Angiogenesis (41) \\
\hline$m i R-21$ and $m i R-1225-5 p$ & NA & Invasion (46) \\
\hline circNRIP1 & AKT1/mTOR pathway & Progression (47) \\
\hline circ-RanGAP1 & miR-877-3p & Invasion and Metastasis (48) \\
\hline
\end{tabular}

BLID, BH3-like motif-containing protein; CDH1, Cadherin-1; EvS, Extracellular vesicles; GC, Gastric cancer; HUVECs, Human umbilical vein endothelial cells; HMGA2, high mobility group AT-hook 2; mTOR, Mammalian target of rapamycin; NA, Not available; PMCs, Peritoneal mesothelial cells; PTEN, Phosphatase and tensin homolog; SUFU, Suppressor of fused protein; SFRP1, Secreted frizzled-related protein 1; VEGF, vascular endothelial growth factor. 
survival of circulating tumor cells after reaching the secondary site (Figure 2).

\section{IMMUNOMODULATION}

The pre-metastasis niche creates a favorable microenvironment for circulating tumor cells to metastasize to specific organs and locations (49). The process of tumor metastasis is closely related to the body's immune system (50). EVs derived from GC act on specific signaling pathways to induce immune cell apoptosis, autophagy, and functional expression to promote tumor progression (51). In the setting of adaptive immunity, Qu et al. demonstrated that GC-derived exosomes could increase PI3K proteasome degradation through the ubiquitin ligase cbl family, inactivate PI3K/Akt signaling, and activate caspase 3, 8, 9 to mediate apoptosis of Jurkat $\mathrm{T}$ cells (52). However, in the innate immune response, GC-derived exosomes induced neutrophils autophagy and promoted tumor activation by acting on $\mathrm{HMGB} 1 / \mathrm{TLR} 4 / \mathrm{NF}-\kappa \mathrm{B}$ signals (53). GC-derived exosomes can induce macrophages to secrete inflammatory factors through the NF- $\mathrm{KB}$ signaling pathway to promote tumor proliferation, migration, and invasion (54). Besides, under the hypoglycemic state, the expression of miR-451 carried by GC-derived exosomes was up-regulated, which promoted Th17 cells differentiation by activating the NF- $\kappa B$ pathway (55). Previous studies have shown that mesenchymal stem cells are also related to immune regulation. When Shen et al. co-cultured mesenchymal stem cells with GC-derived exosomes, they found that mesenchymal stem cells can activate immune cells through the NF- $\kappa \mathrm{B}$ signaling pathway, maintain the function of the inflammatory environment, thereby promoting tumor growth (56). All the aforementioned studies indicate that GC-derived exosomes play a negative regulatory role by acting on immune cells, thereby helping to form the premetastatic niche before metastasis (Supplementary Table 1).

\section{ANGIOGENESIS}

Angiogenesis refers to the process of forming new blood vessels from the original capillaries or post-capillary veins (57). These new capillaries provide the tumor with nutrients, oxygen, and growth factors to promote tumor growth and metastasis (58). GC-derived EVs form a pre-metastatic microenvironment that is conducive to tumor growth by promoting tumor angiogenesis.

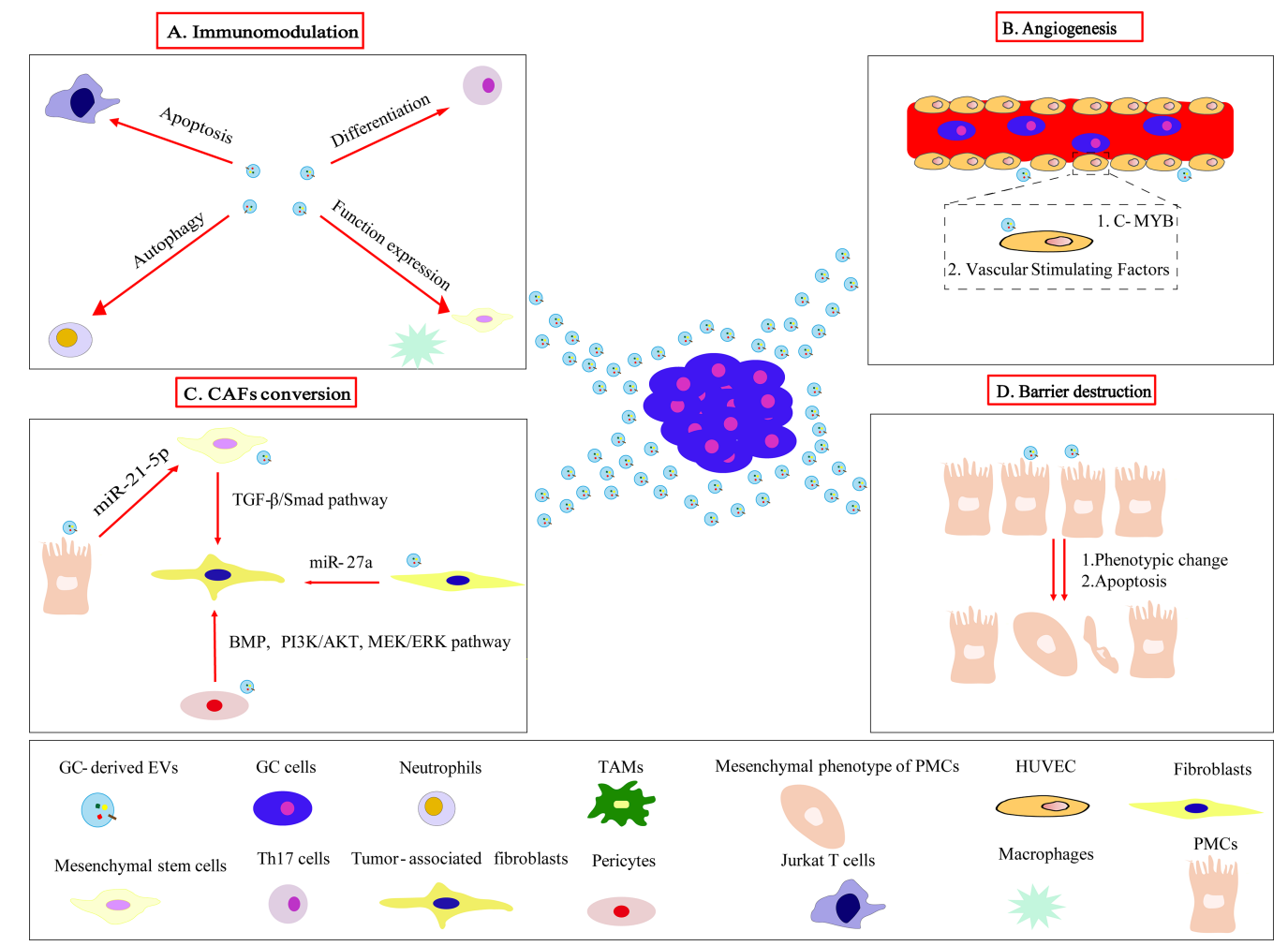

FIGURE 2 | GC-derived EVs acts on target cells to mediate the formation of pre-metastasis niche. (A) GC-derived EVs modulate the immune microenvironment by inducing Jurkat T cells apoptosis, neutrophils autophagy, Th17 cells differentiation, functional expression of macrophages and mesenchymal stem cells, eventually promoting tumor progression and metastasis. (B) GC-derived EVs target C-MYB on vascular endothelial cells and regulate vascular stimulating factors to promote angiogenesis. (C) GC-derived EVs convert fibroblasts, pericytes, mesenchymal stem cells, PMCs to CAFs through different mechanisms. (D) Phenotypic changes and apoptosis of PMCs induced by GC-derived EVs clear the peritoneal mesothelial cell barrier layers. 
Previous studies have shown that C-MYB, as a transcription factor, is closely related to angiogenesis. Researchers have confirmed that human umbilical vein endothelial cells (HUVECs) that overexpressed c-MYB have reduced migration, proliferation, and ring formation. GC-derived exosomes also express miR-130a, which promotes angiogenesis by targeting C-MYB on vascular endothelial cells, whether in vivo or in vitro (41). After the researchers incubated vascular endothelial cells with exosomes derived from irradiated GC cells, the proliferation, mobility, and invasiveness of vascular endothelial cells increased which was counteracted by VEGFR-2 inhibitorApatinib (59). These studies suggest that GC-derived EVs promote vascular endothelial cells proliferation and migration in different ways to achieve angiogenesis.

\section{STROMAL REMODELING}

Stromal cells and extracellular matrix components are the key components of the tumor microenvironment, providing support and nutrition in tumor growth, progression, and metastasis (60). The stromal cells mainly include fibroblasts, inflammatory cells, immune cells, and pericytes. EVs secreted by tumors play a crucial role in stromal cell remodeling by reprogramming fibroblasts, pericytes, immune cells, and by extension, mediating the formation of an extracellular matrix that facilitates the growth of secondary tumor sites, thereby promoting tumor growth, invasion, and metastasis (12).

\section{Cancer-Associated Fibroblasts (CAFs) Conversion}

Among all the stromal cells, CAFs play a crucial role in tumor invasion, progression, and metastasis (61). By secreting a series of chemokines, cytokines, and proteases to participate in matrix remodeling, regulating the recruitment and function of immune cells in the tumor microenvironment; hence creating a niche for cancer cells to promote their malignant tendencies (62). EVs mediate the transformation of stromal cells into CAFs by acting as a conduit between tumor cells and stromal cells (60). Proofs have shown that high and stable expression of miR-27a in exosomes was related to poor clinical prognosis. Similarly, GCderived exosomes mediated the transformation of fibroblasts into CAFs by expressing miR-27a, and have been shown to promote the proliferation and metastasis of cancer cells both in vivo and in vitro (42). Similarly, Wang et al. postulated that GC-derived exosomes can induce macrophages to differentiate into PD1 + tumor-associated macrophages, thereby creating an environment for tumor progression (63). Moreover, GC-derived exosomes can promote the proliferation and migration of pericytes by inducing the transformation of pericytes into CAFs by transferring bone morphogenetic proteins (BMP) and activating PI3K/AKT and MEK/ERK pathways (64). Mesenchymal stem cells have great potential for self-renewal and multi-directional differentiation. Studies have shown that exosomes derived from GC mediate the transformation of human umbilical cord-derived mesenchymal stem cells into CAFs by transferring TGF- $\beta$ and activating TGF- $\beta$ /
Smad pathway (65). It is particularly worth mentioning that PMCs are also one of the important sources of CAFs. On the one hand, when EVs are not involved, the study on peritoneal metastasis has shown that normal mesothelial cells can be converted into CAFs through MMT (66). On the other hand, for EVs, PMCs can be transformed into mesenchymal stem cells first and then into CAFs. GC-derived EVs mediate the transformation of PMCs into mesenchymal cells (67), for example, PMCs can internalize GC-derived exosomes miR-21-5p, and induce PMC MMT by activating the TGF- $\beta /$ Smad pathway via targeting SMAD7 (28). And the mechanism of transition of mesenchymal stem cells to CAFs is as described previously. Besides, another study has shown that EVs derived from malignant ascites may promote the transformation of PMCs to CAFs through TGF- $\beta 1$-induced MMT, and in the EVs-treated group, the phenotypic changes of PMCs under morphological and western blotting assays all indicated a CAFs-like transition (68). Lastly, before metastasis to secondary organs, GC-derived EVs mediate the transition of normal stroma to cancer stroma.

\section{CAFs and Tumor-Associated Macrophages (TAMs) Function}

GC-derived exosomes promote tumor growth, invasion, and metastasis by directly suppressing immune responses and inducing the formation of CAFs and TAMs phenotypes (69). GC-derived EVs promote the transformation of various cells, including fibroblasts, pericytes, PMCs and mesenchymal stem cells, into CAFs with a malignant phenotype (70). Evidence has shown that CAFs promote the migration and invasion of GC cells, and enhance angiogenesis and metastasis that are essential for tumor cells colonization, survival, and growth (71-75). Previous studies have shown that CAFs increased GC cell mobility and invasiveness by up-regulating Rhomboid 5 Homolog 2 (RHBDF2) expression through the TGF- $\beta 1$ signaling pathway (76). Ding et al. showed that CAFs mediate GC angiogenesis through the PI3K/AKT and ERK1/2 signaling pathways through the expression of HGF (77). In addition, CAFs also mediate the invasion and metastasis of GC by acting on multiple signal pathways. For example, Bae's study showed that inhibiting the GAS6/AXL axis can block the interaction between CAFs and GC cells, thereby inhibiting tumor progression (78). CAFs can promote GC in a ligand-independent manner by activating erythropoietin-producing hepatocyte A2 (EphA2) (79). CAFs also activate CXCL12/CXCR4 to promote integrin b1 aggregation and invasiveness in GC (80). Kurashige's research showed that cancer-related epigenetic regulation and inhibition of miR-200b by fibroblasts contributes to cancer infiltration and peritoneal spread of GC (81). Activated GC-associated fibroblasts promote the malignant phenotype and 5-FU of GC through paracrine drug resistance (82). CAFs can promote the malignant degree of GC cells through nodal signaling (83). In addition, previous research has shown that CAFs-derived lumican promotes the progress of GC through the integrin b1FAK signaling pathway (84).

In the pre-metastatic microenvironment, GC-derived EVs mediate the differentiation of local macrophages into 
immunosuppressive M2 macrophages. TAMs promote tumorigenesis via their exosome secretion. Study has shown that TAMs-derived exosomes promote migration of GC cells through the transfer of functional apolipoprotein E (85). In addition, TAMs play an important role in the invasion, development, angiogenesis and metastasis of GC. Evidence has proved that polarized CD163+ TAMs were associated with increased angiogenesis in GC (86). TAMs regulated the invasion and metastasis of GC cells through the TGF 2 / NF- $\kappa \mathrm{B} /$ Kindlin-2 axis (87). TAMs may cause an increase in Bmil expression through $\mathrm{miR}-30 \mathrm{e}$ inhibition, leading to gastrointestinal tumor progression (88). TAMs induced forkhead box Q1 (FOXQ1) expression and promoted GC cell epithelial-mesenchymal transition and metastasis (89). Ding et al. confirmed that CCL5 secreted by TAMs may promote the proliferation, invasion, and metastasis of GC cells through the Stat3 signaling pathway (90). TAMs induce GC invasion and poor prognosis via cyclooxygenase2/MMP9 dependent manner (91). Lastly, macrophages in the peritoneum of GC patients play a supportive role in peritoneal metastasis by producing EGF and VEGF (92). All this evidence highlights that EVs mediate the transformation of normal stroma to cancer stroma, and blocking this transformation is essential for the distant metastasis of tumors.

\section{GC-DERIVED EVs MEDIATED ORGANOPHILIC METASTASIS}

\section{Barrier Destruction and Peritoneal Metastasis (PM)}

Most advanced GC metastasizes to the peritoneum. Therefore, it is crucial to understand the mechanism of PM. Stephen Paget's "seed and soil" hypothesis offers great insight into understanding the mechanism of PM (93). The first line of defense for all abdominal metastasis is the mesothelial cell (94). Recent studies have shown that GC-derived EVs can break through the first barrier of PM by promoting mesothelial cell damage, apoptosis, and phenotypic changes. Deng et al. confirmed that GC-derived exosomes not only induced mesothelial cell apoptosis, but also mediated the transformation of mesothelial cells to mesenchyme, which leads to the destruction of the mesothelial barrier and peritoneal fibrosis, and ultimately promotes PM (67). Likewise, exosomal miR-21-5p induced the mesothelial to mesenchymal transition of peritoneal mesothelial cells by targeting Smad7 and promoted the peritoneal spread of cancer (28).

Research has also revealed that exosomes from malignant ascites in GC patients supported epithelial-mesenchymal transition (EMT) signaling in GC cells in the mouse peritoneal tumor model, thus, resulting in peritoneal tumor cell dissemination (39). Furthermore, the co-culture of exosomes derived from malignant pleural fluid of GC and GC cells can promote tumor migration and increase the expression of mesothelial cell adhesion-related molecules, such as fibronectin 1 and laminin gamma 1 , which may be the mechanism of peritoneal metastasis (95). GC-derived EVs induced PMCs infiltration by activating $\mathrm{Wnt} 3 \mathrm{a} / \beta$-catenin signaling, infiltrating PMCs, and in turn, promoting the subserosal invasion of cancer cells, and mutual attraction between cancer cells and PMCs accelerated tumor invasion which ultimately led to peritoneal metastasis (96). Besides, exosomes derived from GC can mediate peritoneal dissemination via participating in the regulation of signaling pathways. There is evidence that the delivery of miR-106a from GC-derived exosomes plays a vital role in GC peritoneal metastasis via direct regulation of Smad7 (31). Incubation of GC cells with peritoneal mesothelial HMrSV5 cells showed that miR-544 could be transferred from GC-derived EVs to peritoneal cells, where it suppresses the promyelocytic leukemia zinc finger expression and ultimately leads to peritoneal metastasis of GC (97). Zhu et al. proposed that Nicotinamide $\mathrm{N}$-methyltransferase-containing exosomes derived from GC cells could promote peritoneal metastasis via TGF- $\beta /$ smad2 signaling (98).

\section{Liver Metastasis (LM)}

In addition to PM, GC-derived EVs are also involved in the LM of GC. Highly aggressive GC cells can secrete exosomes containing miR-196a-1, which may be related to liver metastasis in vitro and in vivo by targeting SFRP1 (35). In addition, the study also showed that EGFR in exosomes secreted from GC cells can be delivered into the liver and is integrated into the plasma membrane of liver stromal cells, the translocated EGFR effectively activates HGF by inhibiting the expression of $\mathrm{miR}-26 \mathrm{a} / \mathrm{b}$. Secondly, the upregulated paracrine HGF binds to the c-MET receptor on the migrated cancer cells, which was conducive to the formation of a hepato-like microenvironment that promotes liver-specific metastasis (26). In summary, there is increasing evidence on the role of GCderived EVs in organophilic metastasis.

\section{EVs AS BIOMARKERS}

Stable, simple, relatively non-invasive, easy to monitor and follow-up, and tumor specificity are the special advantages of EVs as biomarkers. Previous studies have revealed that exosomal miR-23b can be used as a potential minimally invasive predictive biomarker, suitable for detecting recurrence and prognosis in all GC patients irrespective of stages (99). Previous studies have also demonstrated that exosomal HOXA transcript at the distal tip (HOTTIP) could be a potential biomarker in GC diagnosis and prognosis (100). Recent research has shown that low expression of miR-29b in peritoneal exosomes was associated with postoperative peritoneal recurrence (101). Similarly, a study showed that mesenchymal stem cells derived from GC tissues promoted the progression of GC by transferring exosomal miRNAs to GC cells, thereby providing a potential biomarker for GC (102). Finally, exosomes also promote the progression of GC by expressing proteins. For instance, a previous study showed that the overall survival rate of the high exosomal PDL1 group was significantly lower than the low exosomal PD-L1 group, exosomal PD-L1 in GC patients was negatively associated 
with CD4 T cell and CD8 T-cell count, indicating that exosomal PD-L1 was associated with the immunosuppressive status of GC patients (103). In addition, previous research has shown that CD63 is one of the possible prognostic indicators for patients with GC, since CD63-positive exosomes may be related to the interaction between stromal cells and cancer cells (104).

\section{CONCLUSION}

GC-derived EVs promote the formation of the microenvironment before metastasis through immunomodulation, angiogenesis, stromal cell remodeling, barrier destruction, and organophilic metastasis. Initially, the pre-metastasis microenvironment heralded a lot of excitement in the field of cancer research. With the recent advancement of research, we continue to understand the vital role of EVs in the pre-metastasis microenvironment. At present, the isolation and purification of EVs in vitro are not complicated. Therefore, it should be harnessed as an available and cost-effective potential biomarker in early cancer detection, treatment evaluation, and follow-up. New technologies, including liquid biopsy, are moving the field forward in understanding the mechanisms of tumor metastasis. The latest developments make EVs a promising new field for early diagnosis and detection across cancer types.

\section{REFERENCES}

1. Thrift AP, El-Serag HB. Burden of Gastric Cancer. Clin Gastroenterol Hepatol (2020) 18(3):534-42. doi: 10.1016/j.cgh.2019.07.045

2. Ferlay J, Colombet M, Soerjomataram I, Mathers C, Parkin DM, Pineros M, et al. Estimating the Global Cancer Incidence and Mortality in 2018: GLOBOCAN Sources and Methods. Int J Cancer (2019) 144(8):1941-53. doi: 10.1002/ijc.31937

3. Digklia A, Wagner AD. Advanced Gastric Cancer: Current Treatment Landscape and Future Perspectives. World J Gastroenterol (2016) 22 (8):2403-14. doi: 10.3748/wjg.v22.i8.2403

4. Rawla P, Barsouk A. Epidemiology of Gastric Cancer: Global Trends, Risk Factors and Prevention. Prz Gastroenterol (2019) 14(1):26-38. doi: 10.5114/ pg.2018.80001

5. Paget S. The Distribution of Secondary Growths in Cancer of the Breast. 1889 Cancer Metastasis Rev (1989) 8(2):98-101.

6. Peinado H, Zhang H, Matei IR, Costa-Silva B, Hoshino A, Rodrigues G, et al. Pre-Metastatic Niches: Organ-Specific Homes for Metastases. Nat Rev Cancer (2017) 17(5):302-17. doi: 10.1038/nrc.2017.6

7. Liu Y, Cao X. Characteristics and Significance of the Pre-Metastatic Niche. Cancer Cell (2016) 30(5):668-81. doi: 10.1016/j.ccell.2016.09.011

8. Kosaka N, Yoshioka Y, Fujita Y, Ochiya T. Versatile Roles of Extracellular Vesicles in Cancer. J Clin Invest (2016) 126(4):1163-72. doi: 10.1172/ jci81130

9. Xie C, Ji N, Tang Z, Li J, Chen Q. The Role of Extracellular Vesicles From Different Origin in the Microenvironment of Head and Neck Cancers. Mol Cancer (2019) 18(1):83. doi: 10.1186/s12943-019-0985-3

10. van Niel G, D'Angelo G, Raposo G. Shedding Light on the Cell Biology of Extracellular Vesicles. Nat Rev Mol Cell Biol (2018) 19(4):213-28. doi: 10.1038/nrm.2017.125

11. Bebelman MP, Smit MJ, Pegtel DM, Baglio SR. Biogenesis and Function of Extracellular Vesicles in Cancer. Pharmacol Ther (2018) 188:1-11. doi: 10.1016/j.pharmthera.2018.02.013

\section{AUTHOR CONTRIBUTIONS}

DT was a major writer of the manuscript and designed the tables. GD and SZ conceived and designed the article and guided the selection of references. SL and HS were responsible for figure design and language modification. All the authors read and approved the final manuscript.

\section{FUNDING}

This study was supported by a grant of the National Key R \& D Program of China (No. 2018YFC1313300); six grants from the National Natural Science Foundation of China (No. 81070362, 81172470, 81372629, 81772627, 81874073 \& 81974384); two projects from the Nature Science Foundation of Hunan Province (No. 2021JJ31092 \& 2021JJ31048), and a project from China Cancer Elite Team Innovative Grant (No. 201606).

\section{SUPPLEMENTARY MATERIAL}

The Supplementary Material for this article can be found online at: https://www.frontiersin.org/articles/10.3389/fimmu.2022. 813015/full\#supplementary-material

12. Feng W, Dean DC, Hornicek FJ, Shi H, Duan Z. Exosomes Promote PreMetastatic Niche Formation in Ovarian Cancer. Mol Cancer (2019) 18 (1):124. doi: 10.1186/s12943-019-1049-4

13. Frankel EB, Audhya A. ESCRT-Dependent Cargo Sorting at Multivesicular Endosomes. Semin Cell Dev Biol (2018) 74:4-10. doi: 10.1016/ j.semcdb.2017.08.020

14. Henne WM, Stenmark H, Emr SD. Molecular Mechanisms of the Membrane Sculpting ESCRT Pathway. Cold Spring Harb Perspect Biol (2013) 5(9): a016766. doi: 10.1101/cshperspect.a016766

15. Wollert T, Hurley JH. Molecular Mechanism of Multivesicular Body Biogenesis by ESCRT Complexes. Nature (2010) 464(7290):864-9. doi: $10.1038 /$ nature08849

16. Colombo M, Moita C, van Niel G, Kowal J, Vigneron J, Benaroch P, et al Analysis of ESCRT Functions in Exosome Biogenesis, Composition and Secretion Highlights the Heterogeneity of Extracellular Vesicles. J Cell Sci (2013) 126(Pt 24):5553-65. doi: 10.1242/jcs.128868

17. Stuffers S, Sem Wegner C, Stenmark H, Brech A. Multivesicular Endosome Biogenesis in the Absence of ESCRTs. Traffic (2009) 10(7):925-37. doi: 10.1111/j.1600-0854.2009.00920.x

18. Kagota S, Taniguchi K, Lee SW, Ito Y, Kuranaga Y, Hashiguchi Y, et al Analysis of Extracellular Vesicles in Gastric Juice From Gastric Cancer Patients. Int J Mol Sci (2019) 20(4):953. doi: 10.3390/ijms20040953

19. Huang T, Song C, Zheng L, Xia L, Li Y, Zhou Y. The Roles of Extracellular Vesicles in Gastric Cancer Development, Microenvironment, Anti-Cancer Drug Resistance, and Therapy. Mol Cancer (2019) 18(1):62. doi: 10.1186/ s12943-019-0967-5

20. Liu D, Li C, Trojanowicz B, Li X, Shi D, Zhan C, et al. CD97 Promotion of Gastric Carcinoma Lymphatic Metastasis Is Exosome Dependent. Gastric Cancer (2016) 19(3):754-66. doi: 10.1007/s10120-015-0523-y

21. Li C, Liu DR, Li GG, Wang HH, Li XW, Zhang W, et al. CD97 Promotes Gastric Cancer Cell Proliferation and Invasion Through Exosome-Mediated MAPK Signaling Pathway. World J Gastroenterol (2015) 21(20):6215-28. doi: 10.3748/wjg.v21.i20.6215 
22. Scavo MP, Depalo N, Rizzi F, Ingrosso C, Fanizza E, Chieti A, et al. FZD10 Carried by Exosomes Sustains Cancer Cell Proliferation. Cells (2019) 8 (8):777. doi: 10.3390/cells8080777

23. Scavo MP, Cigliano A, Depalo N, Fanizza E, Bianco MG, Denora N, et al. Frizzled-10 Extracellular Vesicles Plasma Concentration Is Associated With Tumoral Progression in Patients With Colorectal and Gastric Cancer. J Oncol (2019) 2019:2715968. doi: 10.1155/2019/2715968

24. Che Y, Geng B, Xu Y, Miao X, Chen L, Mu X, et al. Helicobacter PyloriInduced Exosomal MET Educates Tumour-Associated Macrophages to Promote Gastric Cancer Progression. J Cell Mol Med (2018) 22(11):570819. doi: $10.1111 / \mathrm{jcmm} .13847$

25. Yen EY, Miaw SC, Yu JS, Lai IR. Exosomal TGF- $\beta 1$ Is Correlated With Lymphatic Metastasis of Gastric Cancers. Am J Cancer Res (2017) 7 (11):2199-208.

26. Zhang H, Deng T, Liu R, Bai M, Zhou L, Wang X, et al. Exosome-Delivered EGFR Regulates Liver Microenvironment to Promote Gastric Cancer Liver Metastasis. Nat Commun (2017) 8:15016. doi: 10.1038/ncomms15016

27. Shi SS, Zhang HP, Yang CQ, Li LN, Shen Y, Zhang YQ. Exosomal miR-155$5 p$ Promotes Proliferation and Migration of Gastric Cancer Cells by Inhibiting TP53INP1 Expression. Pathol Res Pract (2020) 216(6):152986. doi: 10.1016/j.prp.2020.152986

28. Li Q, Li B, Li Q, Wei S, He Z, Huang X, et al. Exosomal miR-21-5p Derived From Gastric Cancer Promotes Peritoneal Metastasis via Mesothelial-toMesenchymal Transition. Cell Death Dis (2018) 9(9):854. doi: 10.1038/ s41419-018-0928-8

29. Du J, Liang Y, Li J, Zhao JM, Wang ZN, Lin XY. Gastric Cancer Cell-Derived Exosomal microRNA-23a Promotes Angiogenesis by Targeting PTEN. Front Oncol (2020) 10:326:326. doi: 10.3389/fonc.2020.00326

30. Piao HY, Guo S, Wang Y, Zhang J. Exosome-Transmitted lncRNA PCGEM1 Promotes Invasive and Metastasis in Gastric Cancer by Maintaining the Stability of SNAI1. Clin Transl Oncol (2021) 23(2):246-56. doi: 10.1007/ s12094-020-02412-9

31. Zhu M, Zhang N, He S, Lu X. Exosomal miR-106a Derived From Gastric Cancer Promotes Peritoneal Metastasis via Direct Regulation of Smad7. Cell Cycle (Georgetown Tex) (2020) 19(10):1200-21. doi: 10.1080/15384101.2020. 1749467

32. Wei S, Peng L, Yang J, Sang H, Jin D, Li X, et al. Exosomal Transfer of miR15b-3p Enhances Tumorigenesis and Malignant Transformation Through the DYNLT1/Caspase-3/Caspase-9 Signaling Pathway in Gastric Cancer. J Exp Clin Cancer Res (2020) 39(1):32. doi: 10.1186/s13046-019-1511-6

33. Deng T, Zhang H, Yang H, Wang H, Bai M, Sun W, et al. Exosome miR-155 Derived From Gastric Carcinoma Promotes Angiogenesis by Targeting the C-MYB/VEGF Axis of Endothelial Cells. Mol Ther Nucleic Acids (2020) 19:1449-59. doi: 10.1016/j.omtn.2020.01.024

34. Zhou Z, Zhang H, Deng T, Ning T, Liu R, Liu D, et al. Exosomes Carrying MicroRNA-155 Target Forkhead Box O3 of Endothelial Cells and Promote Angiogenesis in Gastric Cancer. Mol Ther Oncolytics (2019) 15:223-33. doi: 10.1016/j.omto.2019.10.006

35. Feng C, She J, Chen X, Zhang Q, Zhang X, Wang Y, et al. Exosomal miR196a-1 Promotes Gastric Cancer Cell Invasion and Metastasis by Targeting SFRP1. Nanomed (Lond Engl) (2019) 14(19):2579-93. doi: 10.2217/nnm2019-0053

36. Huang J, Shen M, Yan M, Cui Y, Gao Z, Meng X. Exosome-Mediated Transfer of miR-1290 Promotes Cell Proliferation and Invasion in Gastric Cancer via NKD1. Acta Biochim Biophys Sin (Shanghai) (2019) 51(9):900-7. doi: 10.1093/abbs/gmz077

37. Bai M, Li J, Yang H, Zhang H, Zhou Z, Deng T, et al. miR-135b Delivered by Gastric Tumor Exosomes Inhibits FOXO1 Expression in Endothelial Cells and Promotes Angiogenesis. Mol Ther (2019) 27(10):1772-83. doi: 10.1016/ j.ymthe.2019.06.018

38. Liu X, Lu Y, Xu Y, Hou S, Huang J, Wang B, et al. Exosomal Transfer of miR501 Confers Doxorubicin Resistance and Tumorigenesis via Targeting of BLID in Gastric Cancer. Cancer Lett (2019) 459:122-34. doi: 10.1016/ j.canlet.2019.05.035

39. Hu Y, Qi C, Liu X, Zhang C, Gao J, Wu Y, et al. Malignant Ascites-Derived Exosomes Promote Peritoneal Tumor Cell Dissemination and Reveal a Distinct miRNA Signature in Advanced Gastric Cancer. Cancer Lett (2019) 457:142-50. doi: 10.1016/j.canlet.2019.04.034
40. Yang H, Fu H, Wang B, Zhang X, Mao J, Li X, et al. Exosomal miR-423-5p Targets SUFU to Promote Cancer Growth and Metastasis and Serves as a Novel Marker for Gastric Cancer. Mol Carcinog (2018) 57(9):1223-36. doi: $10.1002 / \mathrm{mc} .22838$

41. Yang H, Zhang H, Ge S, Ning T, Bai M, Li J, et al. Exosome-Derived miR130a Activates Angiogenesis in Gastric Cancer by Targeting C-MYB in Vascular Endothelial Cells. Mol Ther (2018) 26(10):2466-75. doi: 10.1016/ j.ymthe.2018.07.023

42. Wang J, Guan X, Zhang Y, Ge S, Zhang L, Li H, et al. Exosomal miR-27a Derived From Gastric Cancer Cells Regulates the Transformation of Fibroblasts Into Cancer-Associated Fibroblasts. Cell Physiol Biochem Int J Exp Cell Physiol Biochem Pharmacol (2018) 49(3):869-83. doi: 10.1159/ 000493218

43. Pan L, Liang W, Fu M, Huang ZH, Li X, Zhang W, et al. Exosomes-Mediated Transfer of Long Noncoding RNA ZFAS1 Promotes Gastric Cancer Progression. J Cancer Res Clin Oncol (2017) 143(6):991-1004. doi: 10.1007/s00432-017-2361-2

44. Li W, Gao YQ. MiR-217 Is Involved in the Carcinogenesis of Gastric Cancer by Down-Regulating CDH1 Expression. Kaohsiung J Med Sci (2018) 34 (7):377-84. doi: 10.1016/j.kjms.2018.02.003

45. Ohshima K, Inoue K, Fujiwara A, Hatakeyama K, Kanto K, Watanabe Y, et al. Let-7 microRNA Family Is Selectively Secreted Into the Extracellular Environment via Exosomes in a Metastatic Gastric Cancer Cell Line. PloS One (2010) 5(10):e13247. doi: 10.1371/journal.pone.0013247

46. Tokuhisa M, Ichikawa Y, Kosaka N, Ochiya T, Yashiro M, Hirakawa K, et al. Exosomal miRNAs From Peritoneum Lavage Fluid as Potential Prognostic Biomarkers of Peritoneal Metastasis in Gastric Cancer. PloS One (2015) 10 (7):e0130472. doi: 10.1371/journal.pone.0130472

47. Zhang $\mathrm{X}$, Wang $\mathrm{S}$, Wang $\mathrm{H}$, Cao J, Huang $\mathrm{X}$, Chen Z, et al. Circular RNA Circnrip1 Acts as a microRNA-149-5p Sponge to Promote Gastric Cancer Progression via the AKT1/mTOR Pathway. Mol Cancer (2019) 18(1):20. doi: 10.1186/s12943-018-0935-5

48. Lu J, Wang YH, Yoon C, Huang XY, Xu Y, Xie JW, et al. Circular RNA CircRanGAP1 Regulates VEGFA Expression by Targeting miR-877-3p to Facilitate Gastric Cancer Invasion and Metastasis. Cancer Lett (2020) 471:38-48. doi: 10.1016/j.canlet.2019.11.038

49. Guo Y, Ji X, Liu J, Fan D, Zhou Q, Chen C, et al. Effects of Exosomes on PreMetastatic Niche Formation in Tumors. Mol Cancer (2019) 18(1):39. doi: 10.1186/s12943-019-0995-1

50. Gonzalez H, Hagerling C, Werb Z. Roles of the Immune System in Cancer: From Tumor Initiation to Metastatic Progression. Genes Dev (2018) 32(1920):1267-84. doi: 10.1101/gad.314617.118

51. Nicholson LB. The Immune System. Essays Biochem (2016) 60(3):275-301 doi: 10.1042/ebc20160017

52. Qu JL, Qu XJ, Qu JL, Qu XJ, Zhao MF, Teng YE, et al. The Role of Cbl Family of Ubiquitin Ligases in Gastric Cancer Exosome-Induced Apoptosis of Jurkat T Cells. Acta Oncol (2009) 48(8):1173-80. doi: 10.3109/ 02841860903032817

53. Zhang X, Shi H, Yuan X, Jiang P, Qian H, Xu W. Tumor-Derived Exosomes Induce N2 Polarization of Neutrophils to Promote Gastric Cancer Cell Migration. Mol Cancer (2018) 17(1):146. doi: 10.1186/s12943-018-0898-6

54. Wu L, Zhang X, Zhang B, Shi H, Yuan X, Sun Y, et al. Exosomes Derived

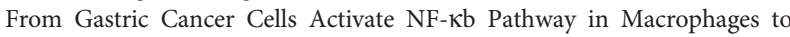
Promote Cancer Progression. Tumour Biol (2016) 37(9):12169-80. doi: 10.1007/s13277-016-5071-5

55. Liu F, Bu Z, Zhao F, Xiao D. Increased T-Helper 17 Cell Differentiation Mediated by Exosome-Mediated microRNA-451 Redistribution in Gastric Cancer Infiltrated T Cells. Cancer Sci (2018) 109(1):65-73. doi: 10.1111/ cas. 13429

56. Shen Y, Xue C, Li X, Ba L, Gu J, Sun Z, et al. Effects of Gastric Cancer CellDerived Exosomes on the Immune Regulation of Mesenchymal Stem Cells by the NF-kB Signaling Pathway. Stem Cells Dev (2019) 28(7):464-76. doi: $10.1089 / \mathrm{scd} .2018 .0125$

57. Viallard C, Larrivée B. Tumor Angiogenesis and Vascular Normalization: Alternative Therapeutic Targets. Angiogenesis (2017) 20(4):409-26. doi: 10.1007/s10456-017-9562-9

58. Aslan C, Maralbashi S, Salari F, Kahroba H, Sigaroodi F, Kazemi T, et al. Tumor-Derived Exosomes: Implication in Angiogenesis and 
Antiangiogenesis Cancer Therapy. J Cell Physiol (2019) 234(10):16885-903. doi: $10.1002 /$ jcp. 28374

59. Li G, Lin H, Tian R, Zhao P, Huang Y, Pang X, et al. VEGFR-2 Inhibitor Apatinib Hinders Endothelial Cells Progression Triggered by Irradiated Gastric Cancer Cells-Derived Exosomes. J Cancer (2018) 9(21):4049-57. doi: $10.7150 /$ jca. 25370

60. Maacha S, Bhat AA, Jimenez L, Raza A, Haris M, Uddin S, et al. Extracellular Vesicles-Mediated Intercellular Communication: Roles in the Tumor Microenvironment and Anti-Cancer Drug Resistance. Mol Cancer (2019) 18(1):55. doi: 10.1186/s12943-019-0965-7

61. Denton AE, Roberts EW, Fearon DT. Stromal Cells in the Tumor Microenvironment. Adv Exp Med Biol (2018) 1060:99-114. doi: 10.1007/ 978-3-319-78127-3_6

62. Chen X, Song E. Turning Foes to Friends: Targeting Cancer-Associated Fibroblasts. Nat Rev Drug Discov (2019) 18(2):99-115. doi: 10.1038/s41573018-0004-1

63. Wang F, Li B, Wei Y, Zhao Y, Wang L, Zhang P, et al. Tumor-Derived Exosomes Induce PD1(+) Macrophage Population in Human Gastric Cancer That Promotes Disease Progression. Oncogenesis (2018) 7(5):41. doi: 10.1038/s41389-018-0049-3

64. Ning X, Zhang H, Wang C, Song X. Exosomes Released by Gastric Cancer Cells Induce Transition of Pericytes Into Cancer-Associated Fibroblasts. Med Sci Monitor Int Med J Exp Clin Res (2018) 24:2350-9. doi: 10.12659/ msm.906641

65. Gu J, Qian H, Shen L, Zhang X, Zhu W, Huang L, et al. Gastric Cancer Exosomes Trigger Differentiation of Umbilical Cord Derived Mesenchymal Stem Cells to Carcinoma-Associated Fibroblasts Through TGF- $\beta /$ Smad Pathway. PloS One (2012) 7(12):e52465. doi: 10.1371/journal.pone.0052465

66. Sandoval P, Jiménez-Heffernan JA, Rynne-Vidal Á, Pérez-Lozano ML, Gilsanz Á, Ruiz-Carpio V, et al. Carcinoma-Associated Fibroblasts Derive From Mesothelial Cells via Mesothelial-to-Mesenchymal Transition in Peritoneal Metastasis. J Pathol (2013) 231(4):517-31. doi: 10.1002/path.4281

67. Deng G, Qu J, Zhang Y, Che X, Cheng Y, Fan Y, et al. Gastric CancerDerived Exosomes Promote Peritoneal Metastasis by Destroying the Mesothelial Barrier. FEBS Lett (2017) 591(14):2167-79. doi: 10.1002/18733468.12722

68. Wei M, Yang T, Chen X, Wu Y, Deng X, He W, et al. Malignant AscitesDerived Exosomes Promote Proliferation and Induce Carcinoma-Associated Fibroblasts Transition in Peritoneal Mesothelial Cells. Oncotarget (2017) 8 (26):42262-71. doi: 10.18632/oncotarget.15040

69. Whiteside TL. Tumor-Derived Exosomes and Their Role in Cancer Progression. Adv Clin Chem (2016) 74:103-41. doi: 10.1016/ bs.acc.2015.12.005

70. Zhang Q, Chai S, Wang W, Wan C, Zhang F, Li Y, et al. Macrophages Activate Mesenchymal Stem Cells to Acquire Cancer-Associated FibroblastLike Features Resulting in Gastric Epithelial Cell Lesions and Malignant Transformation In Vitro. Oncol Lett (2019) 17(1):747-56. doi: 10.3892/ ol.2018.9703

71. Zhang Q, Peng C. Cancer-Associated Fibroblasts Regulate the Biological Behavior of Cancer Cells and Stroma in Gastric Cancer. Oncol Lett (2018) 15 (1):691-8. doi: 10.3892/ol.2017.7385

72. Ham IH, Lee D, Hur H. Role of Cancer-Associated Fibroblast in Gastric Cancer Progression and Resistance to Treatments. J Oncol (2019) 2019:6270784. doi: 10.1155/2019/6270784

73. Yan Y, Wang LF, Wang RF. Role of Cancer-Associated Fibroblasts in Invasion and Metastasis of Gastric Cancer. World J Gastroenterol (2015) 21(33):9717-26. doi: 10.3748/wjg.v21.i33.9717

74. Hu C, Wang Z, Zhai L, Yang M, Shan L, Chai C, et al. Effects of CancerAssociated Fibroblasts on the Migration and Invasion Abilities of SGC-7901 Gastric Cancer Cells. Oncol Lett (2013) 5(2):609-12. doi: 10.3892/ ol.2012.1023

75. Fuyuhiro Y, Yashiro M, Noda S, Matsuoka J, Hasegawa T, Kato Y, et al. Cancer-Associated Orthotopic Myofibroblasts Stimulates the Motility of Gastric Carcinoma Cells. Cancer Sci (2012) 103(4):797-805. doi: 10.1111/ j.1349-7006.2012.02209.x

76. Ishimoto T, Miyake K, Nandi T, Yashiro M, Onishi N, Huang KK, et al. Activation of Transforming Growth Factor Beta 1 Signaling in Gastric Cancer-Associated Fibroblasts Increases Their Motility, via Expression of
Rhomboid 5 Homolog 2, and Ability to Induce Invasiveness of Gastric Cancer Cells. Gastroenterology (2017) 153(1):191-204.e16. doi: 10.1053/ j.gastro.2017.03.046

77. Ding X, Xi W, Ji J, Cai Q, Jiang J, Shi M, et al. HGF Derived From Cancer -Associated Fibroblasts Promotes Vascularization in Gastric Cancer via PI3K/AKT and ERK1/2 Signaling. Oncol Rep (2018) 40(2):1185-95. doi: 10.3892/or.2018.6500

78. Bae CA, Ham IH, Oh HJ, Lee D, Woo J, Son SY, et al. Inhibiting the GAS6/ AXL Axis Suppresses Tumor Progression by Blocking the Interaction Between Cancer-Associated Fibroblasts and Cancer Cells in Gastric Carcinoma. Gastric Cancer (2020) 23(5):824-36. doi: 10.1007/s10120-02001066-4

79. Hong HN, Won YJ, Shim JH, Kim HJ, Han SH, Kim BS, et al. CancerAssociated Fibroblasts Promote Gastric Tumorigenesis Through EphA2 Activation in a Ligand-Independent Manner. J Cancer Res Clin Oncol (2018) 144(9):1649-63. doi: 10.1007/s00432-018-2683-8

80. Izumi D, Ishimoto $\mathrm{T}$, Miyake $\mathrm{K}$, Sugihara $\mathrm{H}$, Eto $\mathrm{K}$, Sawayama $\mathrm{H}$, et al. CXCL12/CXCR4 Activation by Cancer-Associated Fibroblasts Promotes Integrin $\beta 1$ Clustering and Invasiveness in Gastric Cancer. Int J Cancer (2016) 138(5):1207-19. doi: 10.1002/ijc.29864

81. Kurashige J, Mima K, Sawada G, Takahashi Y, Eguchi H, Sugimachi K, et al. Epigenetic Modulation and Repression of miR-200b by Cancer-Associated Fibroblasts Contribute to Cancer Invasion and Peritoneal Dissemination in Gastric Cancer. Carcinogenesis (2015) 36(1):133-41. doi: 10.1093/carcin/bgu232

82. Ma Y, Zhu J, Chen S, Li T, Ma J, Guo S, et al. Activated Gastric CancerAssociated Fibroblasts Contribute to the Malignant Phenotype and 5-FU Resistance via Paracrine Action in Gastric Cancer. Cancer Cell Int (2018) 18:104. doi: 10.1186/s12935-018-0599-7

83. Pang T, Yin X, Luo T, Lu Z, Nie M, Yin K, et al. Cancer-Associated Fibroblasts Promote Malignancy of Gastric Cancer Cells via Nodal Signalling. Cell Biochem Funct (2020) 38(1):4-11. doi: 10.1002/cbf.3446

84. Wang X, Zhou Q, Yu Z, Wu X, Chen X, Li J, et al. Cancer-Associated Fibroblast-Derived Lumican Promotes Gastric Cancer Progression via the Integrin $\beta 1$-FAK Signaling Pathway. Int J Cancer (2017) 141(5):998-1010. doi: $10.1002 /$ ijc. 30801

85. Zheng P, Luo Q, Wang W, Li J, Wang T, Wang P, et al. Tumor-Associated Macrophages-Derived Exosomes Promote the Migration of Gastric Cancer Cells by Transfer of Functional Apolipoprotein E. Cell Death Dis (2018) 9 (4):434. doi: 10.1038/s41419-018-0465-5

86. Park JY, Sung JY, Lee J, Park YK, Kim YW, Kim GY, et al. Polarized CD163+ Tumor-Associated Macrophages Are Associated With Increased Angiogenesis and CXCL12 Expression in Gastric Cancer. Clin Res Hepatol Gastroenterol (2016) 40(3):357-65. doi: 10.1016/j.clinre.2015.09.005

87. Wang Z, Yang Y, Cui Y, Wang C, Lai Z, Li Y, et al. Tumor-Associated Macrophages Regulate Gastric Cancer Cell Invasion and Metastasis Through Tgfß2/NF- Kb/Kindlin-2 Axis. Chin J Cancer Res (2020) 32(1):72-88. doi: $10.21147 /$ j.issn.1000-9604.2020.01.09

88. Sugihara $H$, Ishimoto $T$, Watanabe $M$, Sawayama $H$, Iwatsuki $M$, Baba $Y$, et al. Identification of miR-30 $\mathrm{e}^{\star}$ Regulation of Bmil Expression Mediated by Tumor-Associated Macrophages in Gastrointestinal Cancer. PloS One (2013) 8(11):e81839. doi: 10.1371/journal.pone.0081839

89. Guo J, Yan Y, Yan Y, Guo Q, Zhang M, Zhang J, et al. Tumor-Associated Macrophages Induce the Expression of FOXQ1 to Promote EpithelialMesenchymal Transition and Metastasis in Gastric Cancer Cells. Oncol Rep (2017) 38(4):2003-10. doi: 10.3892/or.2017.5877

90. Ding H, Zhao L, Dai S, Li L, Wang F, Shan B. CCL5 Secreted by Tumor Associated Macrophages may be a New Target in Treatment of Gastric Cancer. BioMed Pharmacother (2016) 77:142-9. doi: 10.1016/j.biopha.2015.12.004

91. Xu J, Yu Y, He X, Niu N, Li X, Zhang R, et al. Tumor-Associated Macrophages Induce Invasion and Poor Prognosis in Human Gastric Cancer in a Cyclooxygenase-2/MMP9-Dependent Manner. Am J Transl Res (2019) 11(9):6040-54.

92. Song H, Wang T, Tian L, Bai S, Chen L, Zuo Y, et al. Macrophages on the Peritoneum Are Involved in Gastric Cancer Peritoneal Metastasis. J Cancer (2019) 10(22):5377-87. doi: 10.7150/jca.31787

93. Wang Z, Chen JQ, Liu JL, Tian L. Issues on Peritoneal Metastasis of Gastric Cancer: An Update. World J Surg Oncol (2019) 17(1):215. doi: 10.1186/ s12957-019-1761-y 
94. Kenny HA, Nieman KM, Mitra AK, Lengyel E. The First Line of IntraAbdominal Metastatic Attack: Breaching the Mesothelial Cell Layer. Cancer Discov (2011) 1(2):100-2. doi: 10.1158/2159-8290.Cd-11-0117

95. Arita T, Ichikawa D, Konishi H, Komatsu S, Shiozaki A, Ogino S, et al. Tumor Exosome-Mediated Promotion of Adhesion to Mesothelial Cells in Gastric Cancer Cells. Oncotarget (2016) 7(35):56855-63. doi: 10.18632/ oncotarget.10869

96. Tanaka M, Kuriyama S, Itoh G, Maeda D, Goto A, Tamiya Y, et al. Mesothelial Cells Create a Novel Tissue Niche That Facilitates Gastric Cancer Invasion. Cancer Res (2017) 77(3):684-95. doi: 10.1158/00085472.Can-16-0964

97. Kong W, Liu X, Yin G, Zheng S, Zhu A, Yu P, et al. Extracellular Vesicle Derived miR-544 Downregulates Expression of Tumor Suppressor Promyelocytic Leukemia Zinc Finger Resulting in Increased Peritoneal Metastasis in Gastric Cancer. Aging (2020) 12(23):24009-22. doi: 10.18632/aging.104082

98. Zhu AK, Shan YQ, Zhang J, Liu XC, Ying RC, Kong WC. Exosomal NNMT From Peritoneum Lavage Fluid Promotes Peritoneal Metastasis in Gastric Cancer. Kaohsiung J Med Sci (2021) 37(4):305-13. doi: 10.1002/kjm2.12334

99. Kumata Y, Iinuma H, Suzuki Y, Tsukahara D, Midorikawa H, Igarashi Y, et al. Exosome-encapsulated microRNA-23b as a Minimally Invasive Liquid Biomarker for the Prediction of Recurrence and Prognosis of Gastric Cancer Patients in Each Tumor Stage. Oncol Rep (2018) 40 (1):319-30. doi: 10.3892/or.2018.6418

100. Zhao R, Zhang Y, Zhang X, Yang Y, Zheng X, Li X, et al. Exosomal Long Noncoding RNA HOTTIP as Potential Novel Diagnostic and Prognostic Biomarker Test for Gastric Cancer. Mol Cancer (2018) 17(1):68. doi: 10.1186/s12943-018-0817-x

101. Ohzawa H, Saito A, Kumagai Y, Kimura Y, Yamaguchi H, Hosoya Y, et al. Reduced Expression of Exosomal Mir-29s in Peritoneal Fluid Is a Useful Predictor of Peritoneal Recurrence After Curative Resection of Gastric
Cancer With Serosal Involvement. Oncol Rep (2020) 43(4):1081-8. doi: 10.3892/or.2020.7505

102. Wang M, Zhao C, Shi H, Zhang B, Zhang L, Zhang X, et al. Deregulated microRNAs in Gastric Cancer Tissue-Derived Mesenchymal Stem Cells: Novel Biomarkers and a Mechanism for Gastric Cancer. Br J Cancer (2014) 110(5):1199-210. doi: 10.1038/bjc.2014.14

103. Fan Y, Che X, Qu J, Hou K, Wen T, Li Z, et al. Exosomal PD-L1 Retains Immunosuppressive Activity and Is Associated With Gastric Cancer Prognosis. Ann Surg Oncol (2019) 26(11):3745-55. doi: 10.1245/s10434019-07431-7

104. Miki Y, Yashiro M, Okuno T, Kuroda K, Togano S, Hirakawa K, et al. Clinico-Pathological Significance of Exosome Marker CD63 Expression on Cancer Cells and Stromal Cells in Gastric Cancer. PloS One (2018) 13(9): e0202956. doi: 10.1371/journal.pone.0202956

Conflict of Interest: The authors declare that the research was conducted in the absence of any commercial or financial relationships that could be construed as a potential conflict of interest.

Publisher's Note: All claims expressed in this article are solely those of the authors and do not necessarily represent those of their affiliated organizations, or those of the publisher, the editors and the reviewers. Any product that may be evaluated in this article, or claim that may be made by its manufacturer, is not guaranteed or endorsed by the publisher.

Copyright (C) 2022 Tang, Liu, Shen, Deng and Zeng. This is an open-access article distributed under the terms of the Creative Commons Attribution License (CC BY). The use, distribution or reproduction in other forums is permitted, provided the original author(s) and the copyright owner(s) are credited and that the original publication in this journal is cited, in accordance with accepted academic practice. No use, distribution or reproduction is permitted which does not comply with these terms. 